\title{
BEAM COMMISSIONING AND FIRST OPERATION OF THE ISAC DTL AT TRIUMF
}

\author{
R.E. Laxdal, G. Dutto, K. Fong, G. Mackenzie, M. Pasini, R. Poirier, \\ R. Ruegg, TRIUMF, 4004 Wesbrook Mall, Vancouver, Canada
}

\begin{abstract}
The post-accelerator for the ISAC radioactive beam facility combines a $35.4 \mathrm{MHz}$ RFQ and a variable energy $106 \mathrm{MHz}$ DTL. The ISAC DTL is designed to accelerate ions with $3 \leq A / q \leq 6$ to energies fully variable from 0.153 to $1.53 \mathrm{MeV} / \mathrm{u}$. The unique separated function design allows efficient room-temperature acceleration and full energy variability with no degradation in beam quality. The first stable beams were accelerated in the DTL in Dec. 2000 with full commissioning in the first months of 2001. Test results with stable beam will be reported and compared to beam simulation results. In addition the initial operating experience with stable beam delivery is reported.
\end{abstract}

\section{INTRODUCTION}

The post-accelerator for the ISAC radioactive ion beam facility[1] includes a $35.4 \mathrm{MHz}$ RFQ to accelerate beams of $A / q \leq 30$ from $2 \mathrm{keV} / \mathrm{u}$ to $153 \mathrm{keV} / \mathrm{u}$ and a post-stripper, 106.1 MHz variable energy drift tube linac (DTL) to accelerate ions of $3 \leq A / q \leq 6$ to a final energy from 0.15 to $1.53 \mathrm{MeV} / \mathrm{u}$. The RFQ commissioning has been reported previously[2, 3].

The DTL has been configured as a separated function linac. [4]. Five independently phased IH tanks operating at $\phi_{s}=0^{\circ}$ provide the main acceleration. Longitudinal focussing is provided by independently phased, triple gap, split-ring resonator structures[5] positioned before the second, third and fourth IH tanks. Quadrupole triplets placed after each IH tank maintain transverse focussing. To achieve a reduced final energy, the higher energy IH tanks are turned off starting at the downsteam end and the voltage and phase in the last operating tank is varied. This unique configuration offers an efficient roomtemperature approach to a variable energy cw heavy ion linac. A schematic drawing of the DTL is shown in Fig. 1.

In April 2000 the first complete sub-section of the DTL consisting of the first IH tank, Tank1, plus a quadrupole triplet, Triplet1, and triple gap buncher, Buncher1, was installed and tested with beam prior to the installation of the remaining four sub-systems in late 2000 (Fig. 2) with the first beam accelerated to full ISAC energy of $1.53 \mathrm{MeV} / \mathrm{u}$ on Dec. $21 / 2000$.

\section{BEAM DYNAMICS}

At full voltage the beam dynamics are typical of a $0^{\circ}$ accelerating structure[6]; matched beams enter each accelerating section converging in all dimensions and after
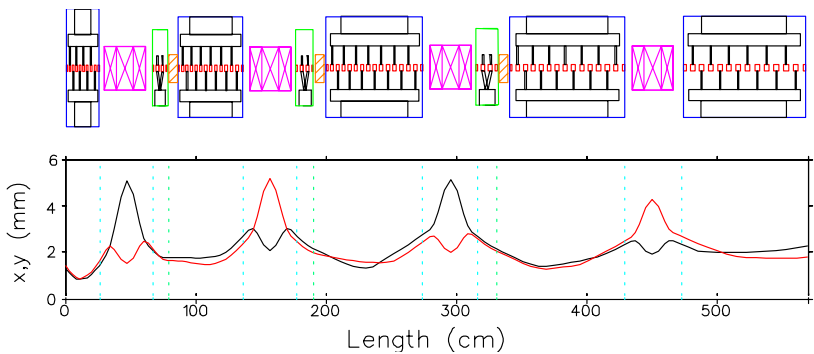

Figure 1: Schematic drawing of the ISAC variable energy $106 \mathrm{MHz}$ DTL (upper figure) and corresponding beam envelopes (lower figure). The beam envelopes define the $x$ and $y$ maximum half sizes of the beam for matched elliptical emittances of $0.25 \pi \mu \mathrm{m}$ (normalized).

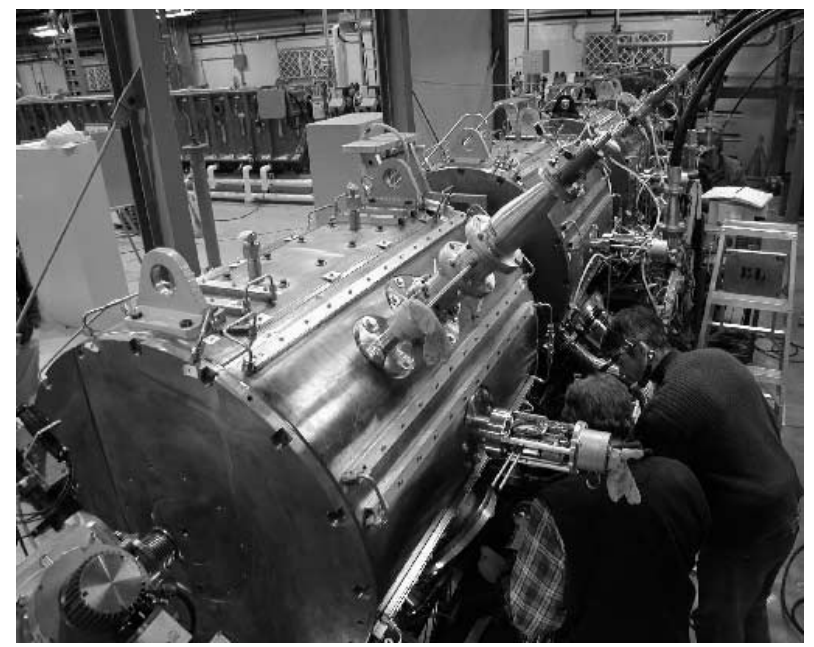

Figure 2: The ISAC $106 \mathrm{MHz}$ DTL.

acceleration the now diverging beams are refocussed before the next accelerating section. In order to maximize longitudinal acceptance the intertank length is minimized. Each triplet consists of quadrupoles with effective lengths of 5.8, 8.7 and $5.8 \mathrm{~cm}$ with maximum gradients of 6.7, 7.5, $6.7 \mathrm{~T} / \mathrm{m}$ and a total length of $37.5 \mathrm{~cm}$. Horizontal and vertical steering coils are wound in the central quadrupole.

At a reduced tank voltage the particle bunch is phased negatively with respect to the synchronous phase so that as the particles lose step with the synchronous particle and drift to more positive phases they gain the required energy. The upstream buncher is used to match the beam to the detuned tank. The buncher following the tank is then used to capture the diverging beam. In Fig. 3 we show the ini- 
tial and final position of a grid of particles in longitudinal phase space after simulated acceleration in Tank1 for two different relative tank voltages, $V / V_{o}=0.4$ and 0.8. Distortion of phase space occurs for phases near $0^{\circ}$. Below this the energy gain falls off nearly linearly with phase. Fig. 5 summarizes voltage and phase set-points for DTL tanks established through beam simulations.
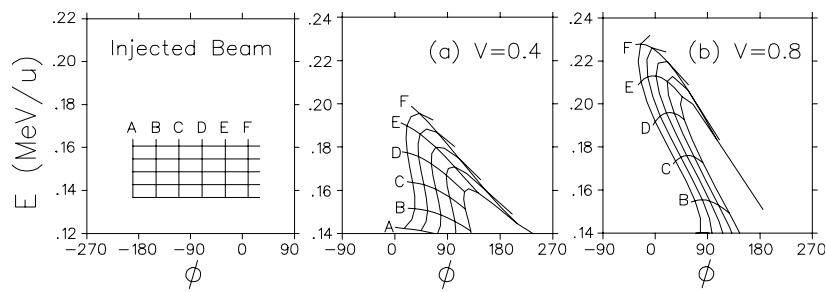

Figure 3: Final position in $E-\phi$ space of an initial grid of particles after acceleration through Tank1 for relative voltages of 0.4 and 0.8 with respect to the full energy case.

\section{TEST SET-UP}

Beam tests have been completed both for Tank1 alone[7] and for the full DTL. A schematic of the test set-up for the full DTL is shown in Fig. 4. The medium energy beam

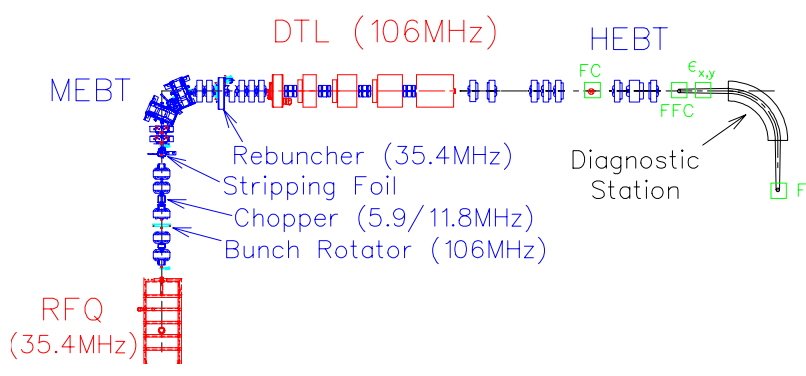

Figure 4: A schematic view of the MEBT, DTL and diagnostic station.

transport (MEBT) transports the beam between RFQ and DTL. The MEBT consists of a matching section for the stripping foil, a charge selection section and a final matching section before the DTL. This matching section includes a $35.4 \mathrm{MHz}$ spiral buncher and four quadrupoles. Narrow diagnostic boxes upstream of both the MEBT rebuncher and each DTL IH-Tank each house a profile monitor and Faraday cup.

The diagnostic station downstream of the DTL includes Faraday cups (FC) for beam transmission measurements, a slit and harp transverse emittance rig $\left(\epsilon_{x, y}\right)$, a $50 \Omega$ co-axial fast Faraday cup (FFC) for pulse width and TOF measurements and a $90^{\circ}$ bending magnet with object and image slits and dispersion of $3 \mathrm{~cm} / \%\left(\frac{\Delta p}{p}\right)$ to analyze the energy and energy spread in the beam.

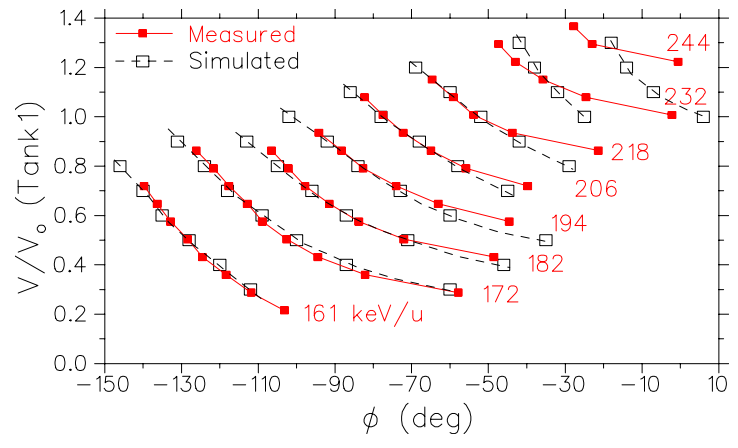

Figure 5: Resultant final energy contours as a function of Tank1 voltage and phase; the small squares (solid lines) correspond to measured values and the large squares (dashed lines) correspond to simulated values.

\section{BEAM TEST RESULTS}

The transverse emittances injected into the DTL are typically $0.06 \pi \mu \mathrm{m}$ (normalized). The longitudinal emittance is measured to be (95\% included) $0.6 \pi \mathrm{keV} / \mathrm{u}-\mathrm{ns}$ for the unstripped beam and about two to three times this for the stripped beam.

\subsection{TANK 1 TESTS}

Beam energy and pulse width measurements for various Tank1 voltage and phase values (Buncher1 off) confirm that variable energy operation works as predicted. Measured Tank1 voltage and phase values to achieve a given energy are plotted as solid contours in Fig. 5 and compared to simulation results (dashed lines through large open squares).Agreement is good except at the high voltage/low phase regime where some discrepancy exists that requires further investigation.

Longitudinal emittance measurements of the accelerated beam are done by varying the Buncher1 voltage and measuring the energy and time spectra at an energy spread minumum. These spectra give emittance values of $0.5-$ $0.6 \pi \mathrm{keV} / \mathrm{u}-\mathrm{ns}$ consistent with little or no longitudinal emittance growth over the Tank1 energy range.

Transverse emittance measurements were completed for Tank 1 operation with ${ }^{14} \mathrm{~N}^{4+}$ and are summarized in Table 1. Listed are computed normalized emittances (4RMS values) before the RFQ, after RFQ acceleration and stripping, after rebunching in MEBT, after acceleration to full energy in Tank1 and finally after rebunching in Buncher1. The largest emittance increase occurs in the stripping foil with only a small increase during acceleration.

\subsection{FULL DTL BEAM TESTS}

Beam commissioning in Jan. 2001 with the complete DTL using ${ }^{4} \mathrm{He}^{1+}$ beams has established the DTL rf parameters and beam optics settings for over twenty different energy set-points covering the full specified operation range. Initial phase and amplitude set points are deter- 
Table 1: Measured emittances of a ${ }^{14} \mathrm{~N}^{4+}$ beam for different cases.

\begin{tabular}{|c|c|c|c|}
\hline Description & $\mathrm{E}$ & $\beta \epsilon_{x}$ & $\beta \epsilon_{y}$ \\
\hline \hline & $(\mathrm{keV} / \mathrm{u})$ & $(\mathrm{mm}-\mathrm{mrad})$ & $(\mathrm{mm}-\mathrm{mrad})$ \\
\hline LEBT & 2 & $0.021 \pi$ & $0.021 \pi$ \\
\hline After Stripping & 153 & $0.059 \pi$ & $0.072 \pi$ \\
\hline After MEBT35 & 153 & $0.068 \pi$ & $0.074 \pi$ \\
\hline After Tank1 & 200 & $0.084 \pi$ & $0.082 \pi$ \\
\hline After Tank1 & 215 & $0.081 \pi$ & $0.078 \pi$ \\
\hline After Tank1 & 231 & $0.077 \pi$ & $0.075 \pi$ \\
\hline After Buncher1 & 231 & $0.077 \pi$ & $0.088 \pi$ \\
\hline \hline
\end{tabular}

mined empirically by beam energy measurement; each accelerator component is turned on and optimized sequentially before advancing to the next device. The DTL triplets are adjusted to optimize transmission with each large energy step. A summary of the rf system performance is given in another contribution.[8].

The transmission through the DTL was over $95 \%$ for all cases. A summary plot of the accelerated beams is given in Fig. 6.
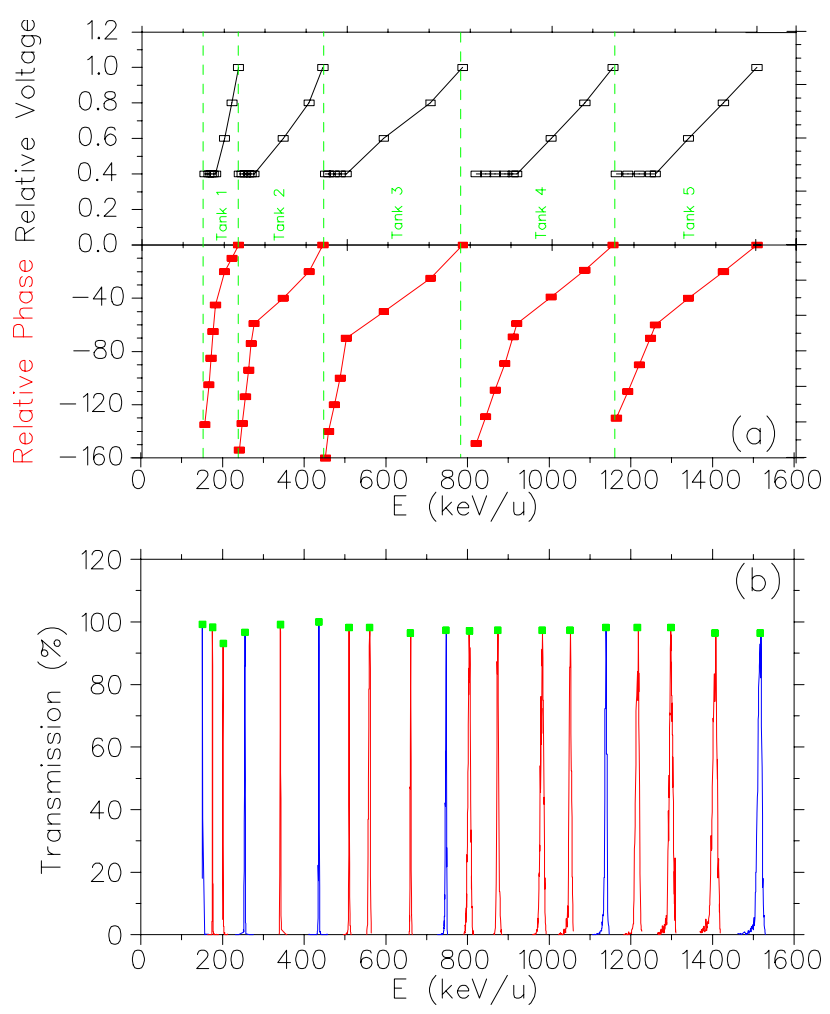

Figure 6: Shown in (a) are the DTL rf set-points established through beam simulation. In (b) we plot the measured transmission for the various beams that have been accelerated through the DTL during commissioning studies with ${ }^{4} \mathrm{He}$.

Longitudinal beam quality for all beams is as predicted. Sample spectra and associated pulse width $5 \mathrm{~m}$ downstream of the DTL are given in Fig. 7.
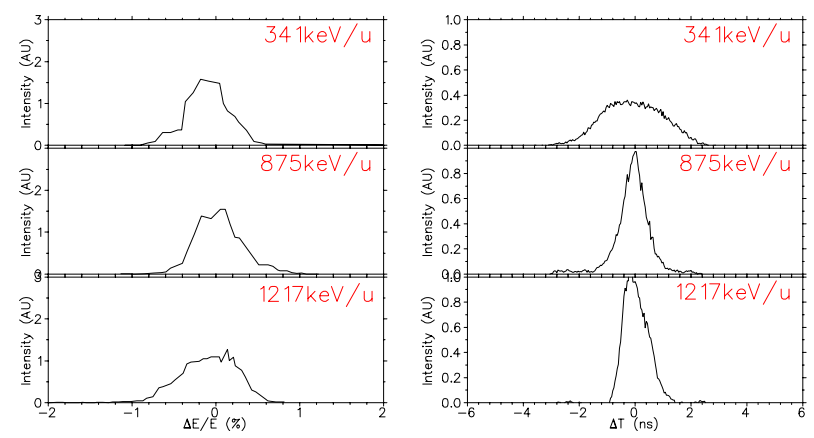

Figure 7: A sample of energy spectra and associated pulse width measured $5 \mathrm{~m}$ from the DTL.

\section{OPERATING EXPERIENCE}

Stable beams of ${ }^{4} \mathrm{He}^{1+},{ }^{14,15} \mathrm{~N}^{4+},{ }^{16} \mathrm{O}^{4+},{ }^{21} \mathrm{Ne}^{5+}$ and ${ }^{24} \mathrm{Mg}^{6+}$ have been delivered to two experimental facilities, the DRAGON recoil mass spectrometer and the TUDA multi-purpose scattering detector, at various beam energies.

Although the ISAC DTL is a variable energy device it still essentially operates as a fixed velocity linac (except for the last operating tank) so that phase relationships between cavities are fixed regardless of particle mass and charge and only the voltage need be scaled, shortening beam tuning time. Small energy steps down to $0.1 \%$ are easily realized by linear voltage or phase steps in the last operating DTL tank. Beam tunes are established by a beam physicist with 24 hour/day delivery by ISAC operations staff. Operators now handle routine procedures such as foil changes, small energy changes at experimenters request and reestablishing beam delivery after hardware trips.

\section{REFERENCES}

[1] R.E. Laxdal, et al, "Completion and Operations of ISAC-I and Extension to ISAC-II", these proceedings.

[2] R. Poirier, et al, "CW Performance of the TRIUMF 8 Meter Long RFQ for Exotic Ions", Proceedings of LINAC2000, Monterey, USA.

[3] R.E. Laxdal, et al, "Beam Test Results with the ISAC $35 \mathrm{MHz}$ RFQ", Proc. of the 1999 Part. Acc. Conf., New York.

[4] R.E. Laxdal, et al, "A Separated Function Drift Tube Linac for the ISAC Project at TRIUMF", Proc. of the 1997 Part. Acc. Conf., Vancouver, Canada, 1997.

[5] A. Vasyuchenko, et al, "Development, Fabrication, and Test of Buncher for the TRIUMF ISAC Facility", these proceedings.

[6] U. Ratzinger, "Interdigital IH Structures", Proc. 1990 Linear Accel. Conf., Los Alamos 1990.

[7] R.E. Laxdal, et al, "First Beam Test with the ISAC Separated Function DTL", Proc. of the XXth Linac Conf. (LINAC2000), Monterey, USA, Aug. 2000.

[8] A. Mitra, et al, "RF Measurement Summary of ISAC DTL Tanks and DTl Bunchers", these proceedings. 